\title{
GROUP METHOD ANALYSIS OF TWO-DIMENSIONAL PLATE IN HEAT FLUX
}

\author{
MINA B. ABD-EL-MALEK, FAYEZ H. MICHAEL, and SAMY M. A. EL-MANSI
}

Received 24 June 2002

\begin{abstract}
The group transformation theoretic approach is applied to present an analytic study of the temperature distribution in a triangular plate, $\Omega$, placed in the field of heat flux, along one boundary, in a form of polynomial functions of any degree " $n$." The Laplace's equation has been reduced to second-order linear ordinary differential equation with an appropriate boundary conditions. Exact solution has been obtained for general shape of $\Omega$ and different boundary conditions.
\end{abstract}

2000 Mathematics Subject Classification: 58J35, 54H15.

1. Introduction. The Laplace's equation arises in many branches of physics, from which it attracts a wide band of researchers. Electrostatic potential, temperature in the case of steady state heat conduction, velocity potential in the case of steady irrotational flow of an ideal fluid, concentration of a substance that is diffusing through a solid, and the displacements of a two-dimensional membrane in equilibrium state are counter examples in which the Laplace's equation is satisfied.

The mathematical technique used in the present analysis is the parametergroup transformation. The group methods, as a class of methods lead to the reduction of the number of independent variables, were first introduced by Birkhoff [6] in 1948, where he made use of one-parameter transformation groups. In 1952, Morgan [9] presented a theory which has led to improvements over earlier similarity methods. The method has been applied intensively by Abd-el-Malek et al. [1, 2, 3, 4, 5, 7].

In this paper, we present a general procedure for applying one-parameter group transformation to the Laplace's equation in a triangular domain. Under the transformation, the partial differential equation with boundary conditions in polynomial form, of any degree, is reduced to an ordinary differential equation with the appropriate corresponding conditions. The equation is then solved analytically for the general form of the triangular domain and boundary conditions.

2. Mathematical formulation. The governing equation for the distribution of temperature $T(x, y)$ is given by

$$
\frac{\partial^{2} T}{\partial x^{2}}+\frac{\partial^{2} T}{\partial y^{2}}=0, \quad(x, y) \in \Omega
$$




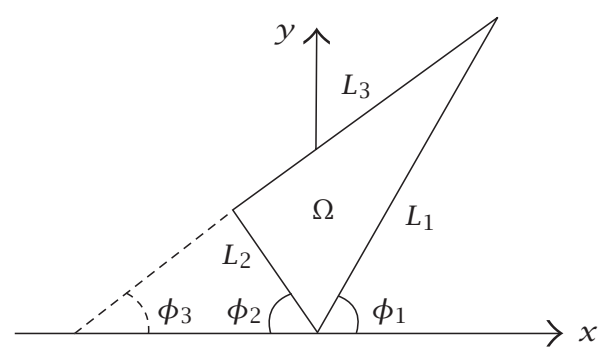

FIGURE 2.1. Geometrical configuration of the problem.

with the following boundary conditions:

$$
\begin{aligned}
T(x, y) & =\alpha q(x), \quad(x, y) \in L_{1}, \\
\frac{\partial T}{\partial y} & =\beta x^{n}, \quad(x, y) \in L_{2} .
\end{aligned}
$$

It is required to find the distribution of the temperature $T(x, y)$ inside the domain $\Omega$, defined in Figure 2.1, and the heat flux across $L_{3}$, where

$$
\begin{aligned}
& L_{1}: y=x \tan \Phi_{1} \\
& L_{2}: y=-x \tan \Phi_{2} \\
& L_{3}: y=x \tan \Phi_{3}+b, \quad b \neq 0, n \in\{0,1,2,3, \ldots\}, \alpha \text { and } \beta \text { are constants. }
\end{aligned}
$$

Write

$$
T(x, y)=w(x, y) q(x), \quad q(x) \not \equiv 0 \text { in } \Omega,
$$

by which differential equation (2.1) takes the form

$$
q(x)\left(\frac{\partial^{2} w}{\partial x^{2}}+\frac{\partial^{2} w}{\partial y^{2}}\right)+2 \frac{\partial w}{\partial x} \frac{d q}{d x}+w \frac{d^{2} q}{d x^{2}}=0,
$$

and the boundary conditions (2.2) take the form

$$
\begin{aligned}
& w(x, y)=\alpha, \quad(x, y) \in L_{1}, \\
& \frac{\partial w}{\partial y}=\frac{\beta x^{n}}{q(x)}, \quad(x, y) \in L_{2} .
\end{aligned}
$$

3. Solution of the problem. The method of solution depends on the application of a one-parameter group transformation to the partial differential equation (2.1). Under this transformation, the two independent variables will be reduced by one and the differential equation (2.1) transforms into an ordinary differential equation in only one independent variable, which is the similarity variable. 
3.1. The group systematic formulation. The procedure is initiated with the group $G$, a class of transformation of one-parameter " $a$ " of the form

$$
G: \bar{S}=C^{S}(a) S+K^{S}(a),
$$

where $S$ stands for $x, y, w$, and $q$ and the $C$ 's and $K$ 's are real valued and at least differentiable in the real argument " $a$."

3.2. The invariance analysis. To transform the differential equation, transformations of the derivatives of $w$ and $q$ are obtained from $G$ via chain-rule operations

$$
\bar{S}_{\bar{i}}=\left(\frac{C^{S}}{C^{i}}\right) S_{i}, \quad \bar{S}_{\overline{i j}}=\left(\frac{C^{S}}{C^{i} C^{j}}\right) S_{i j}, \quad i=x, y ; j=x, y,
$$

where $S$ stands for $w$ and $q$.

Equation (2.5) is said to be invariantly transformed, for some function $H_{1}(a)$, whenever

$$
\bar{q}\left(\bar{w}_{\bar{x} \bar{x}}+\bar{w}_{\bar{y} \bar{y}}\right)+2 \bar{w}_{\bar{x}} \bar{q}_{\bar{x}}+\bar{w} \bar{q}_{\bar{x} \bar{x}}=H_{1}(a)\left[q\left(w_{x x}+w_{y y}\right)+2 w_{x} q_{x}+w q_{x x}\right] .
$$

Substitution from (3.1) into (3.3) yields

$$
\begin{aligned}
& q\left(\left[\frac{C^{q} C^{w}}{\left(C^{x}\right)^{2}}\right] w_{x x}+\left[\frac{C^{q} C^{w}}{\left(C^{y}\right)^{2}}\right] w_{y y}\right) \\
& \quad+2\left[\frac{C^{q} C^{w}}{\left(C^{x}\right)^{2}}\right] w_{x} q_{x}+\left[\frac{C^{q} C^{w}}{\left(C^{x}\right)^{2}}\right] w q_{x x}+\zeta_{1}(a) \\
& =H_{1}(a)\left[q\left(w_{x x}+w_{y y}\right)+2 w_{x} q_{x}+w q_{x x}\right],
\end{aligned}
$$

where

$$
\zeta_{1}(a)=\left(K^{q} C^{w}\right)\left(\frac{w_{x x}}{\left(C^{x}\right)^{2}}+\frac{w_{y y}}{\left(C^{y}\right)^{2}}\right)+\left[\frac{K^{w} C^{q}}{\left(C^{x}\right)^{2}}\right] q_{x x} .
$$

The invariance of (3.4) implies that $\zeta_{1}(a) \equiv 0$. This is satisfied by putting

$$
K^{q}=K^{w}=0, \quad\left[\frac{C^{q} C^{w}}{\left(C^{x}\right)^{2}}\right]=\left[\frac{C^{q} C^{w}}{\left(C^{y}\right)^{2}}\right]=H_{1}(a),
$$

which yields

$$
C^{x}=C^{y}
$$

Moreover, the boundary conditions (2.6) are also invariant in form, implying that

$$
K^{x}=K^{q}=K^{w}=0, \quad C^{q} C^{w}=\left(C^{x}\right)^{n+1}, \quad C^{w}=1 .
$$


Finally, we get the one-parameter group $G$ which transforms invariantly the differential equation (2.5) and the boundary conditions (2.6). The group $G$ is of the form

$$
G:\left\{\begin{array}{l}
\bar{x}=C^{x} x, \\
\bar{y}=C^{x} y+K^{y}, \\
\bar{w}=w, \\
\bar{q}=\left[\left(C^{x}\right)^{n+1}\right] q .
\end{array}\right.
$$

3.3. The complete set of absolute invariants. Our aim is to make use of group methods to represent the problem in the form of an ordinary differential equation. Then, we have to proceed in our analysis to obtain a complete set of absolute invariants.

If $\eta \equiv \eta(x, y)$ is the absolute invariant of the independent variables, then

$$
g_{j}(x, y ; w, q)=F_{j}[\eta(x, y)] ; \quad j=1,2
$$

are the two absolute invariants corresponding to $w$ and $q$. The application of a basic theorem in group theory, see [8], states that a function $g(x, y ; w, q)$ is an absolute invariant of a one-parameter group if it satisfies the following first-order linear differential equation:

$$
\sum_{i=1}^{4}\left(\alpha_{i} S_{i}+\beta_{i}\right) \frac{\partial g}{\partial S_{i}}=0,
$$

where $S_{i}$ stands for $x, y, w$, and $q$, respectively, and

$$
\alpha_{i}=\frac{\partial C^{S_{i}}}{\partial a}\left(a^{0}\right), \quad \beta_{i}=\frac{\partial K^{S_{i}}}{\partial a}\left(a^{0}\right), \quad i=1,2,3,4
$$

and $a^{0}$ denotes the value of " $a$ " which yields the identity element of the group. From (3.6), (3.7), (3.8), and (3.12), we get

$$
\alpha_{1}=\alpha_{2}, \quad \alpha_{3}=0, \quad \beta_{1}=\beta_{3}=\beta_{4}=0
$$

We take $\beta_{2}=0$.

Owing to (3.11), $\eta(x, y)$ is an absolute invariant if it satisfies

$$
x \frac{\partial \eta}{\partial x}+y \frac{\partial \eta}{\partial y}=0
$$

which has a solution in the form

$$
\eta(x, y)=\frac{y}{x} .
$$


To obtain the absolute invariant of the dependent variables $w$ and $q$, apply (3.11), we get

$$
q(x)=R(x) \theta(\eta)
$$

Since $q(x)$ and $R(x)$ are independent of $y$, while $\eta$ is a function of $x$ and $y$, then $\theta(\eta)$ must be a constant, say $\theta(\eta)=1$, hence

$$
q(x)=R(x)
$$

and the second absolute invariant is

$$
w(x, y)=F(\eta)
$$

4. The reduction to an ordinary differential equation. As the general analysis proceeds, the established forms of the dependent and the independent absolute invariant are used to obtain an ordinary differential equation. Generally, the absolute invariant $\eta(x, y)$ has the form given in (3.15).

Substituting from (3.15) and (3.18) into (2.5) yields

$$
\left(\eta^{2}+1\right) \frac{d^{2} F}{d \eta^{2}}-2 \eta\left[\frac{x}{R} \frac{d R}{d x}-1\right] \frac{d F}{d \eta}+\left[\frac{x^{2}}{R} \frac{d^{2} R}{d x^{2}}\right] F=0
$$

For (4.1) to be reduced to an expression in the single independent invariant $\eta$, the coefficients should be constants or functions of $\eta$ alone. Thus,

$$
\begin{aligned}
\frac{x}{R} \frac{d R}{d x} & =C_{1}, \\
\frac{x^{2}}{R} \frac{d^{2} R}{d x^{2}} & =C_{2} .
\end{aligned}
$$

It follows then that, from (4.2),

$$
R(x)=C_{3} x^{C_{1}}
$$

Also, from (4.2) and (4.3), we can show that

$$
C_{2}=C_{1}\left(C_{1}-1\right)
$$

Take $C_{3}=1$ and $C_{1}=n+1$, we get $C_{2}=n(n+1)$, hence we have

$$
\left(\eta^{2}+1\right) \frac{d^{2} F}{d \eta^{2}}-2 \eta n \frac{d F}{d \eta}+n(n+1) F=0
$$


Under the similarity variable $\eta$, the boundary conditions are

$$
F\left(\tan \phi_{1}\right)=\alpha, \quad \frac{d F}{d \eta}\left(-\tan \phi_{2}\right)=\beta
$$

such that the boundary $L_{1}$ or $L_{2}$ does not coincide with the vertical axis.

5. Analytical solution. The solution corresponds to $n \geq 0$ is

$$
\begin{aligned}
F(\eta)= & b_{0} \sum_{k=0}^{[(n+1) / 2]}(-1)^{k}\left(\begin{array}{c}
n+1 \\
2 k
\end{array}\right) \eta^{2 k} \\
& +\left(\frac{b_{1}}{n+1}\right) \sum_{k=0}^{[n / 2]}(-1)^{k}\left(\begin{array}{c}
n+1 \\
2 k+1
\end{array}\right) \eta^{2 k+1}
\end{aligned}
$$

hence we get

$$
\begin{aligned}
T(x, y)= & b_{0} \sum_{k=0}^{[(n+1) / 2]}(-1)^{k}\left(\begin{array}{c}
n+1 \\
2 k
\end{array}\right) y^{2 k} x^{n-2 k+1} \\
& +\left(\frac{b_{1}}{n+1}\right) \sum_{k=0}^{[n / 2]}(-1)^{k}\left(\begin{array}{c}
n+1 \\
2 k+1
\end{array}\right) y^{2 k+1} x^{n-2 k}
\end{aligned}
$$

The heat flux across $L_{3}$ is

$$
\left.\frac{\partial T}{\partial n}(x, y)\right|_{L_{3}}=-\frac{\partial T}{\partial x} \sin \phi_{3}+\frac{\partial T}{\partial y} \cos \phi_{3}
$$

hence we get

$$
\begin{aligned}
\left.\frac{\partial T}{\partial n}(x, y)\right|_{L_{3}}= & b_{0}\left[-\sin \phi_{3} M_{0,1}+\cos \phi_{3} M_{0,2}\right] \\
& +\left(\frac{b_{1}}{n+1}\right)\left[-\sin \phi_{3} M_{1,1}+\cos \phi_{3} M_{1,2}\right]
\end{aligned}
$$

Applying the boundary conditions (4.7), we get

$$
\begin{aligned}
& \alpha=b_{0} z_{0,1}+\left(\frac{b_{1}}{n+1}\right) z_{1,1}, \\
& \beta=b_{0} z_{0,2}+\left(\frac{b_{1}}{n+1}\right) z_{1,2},
\end{aligned}
$$


where

$$
\begin{aligned}
M_{0,1}= & \sum_{k=0}^{[n / 2]}(-1)^{k}(n-2 k+1)\left(\begin{array}{c}
n+1 \\
2 k
\end{array}\right) y^{2 k} x^{n-2 k}, \\
M_{1,1}= & \sum_{k=0}^{[(n-1) / 2]}(-1)^{k}(n-2 k+1)\left(\begin{array}{c}
n+1 \\
2 k+1
\end{array}\right) y^{2 k+1} x^{n-2 k-1}, \\
M_{0,2}= & \sum_{k=1}^{[(n+1) / 2]}(-1)^{k}(2 k)\left(\begin{array}{c}
n+1 \\
2 k
\end{array}\right) y^{2 k-1} x^{n-2 k+1}, \\
M_{1,2}= & \sum_{k=0}^{[n / 2]}(-1)^{k}(2 k+1)\left(\begin{array}{c}
n+1 \\
2 k+1
\end{array}\right) y^{2 k} x^{n-2 k}, \\
z_{0,1}= & \sum_{k=0}^{[(n+1) / 2]}(-1)^{k}\left(\begin{array}{c}
n+1 \\
2 k
\end{array}\right) \tan ^{2 k} \phi_{1}, \\
z_{1,1}= & \sum_{k=0}^{[(n+1) / 2]}(-1)^{k}\left(\begin{array}{c}
n+1 \\
2 k+1
\end{array}\right) \tan ^{2 k+1} \phi_{1}, \\
z_{0,2}= & \sum_{k=0}^{[(n+1) / 2]}(-1)^{k}(2 k)\left(\begin{array}{c}
n+1 \\
2 k
\end{array}\right) \tan ^{2 k-1} \phi_{2}, \\
z_{1,2}= & \sum_{k=0}^{[n / 2]}(-1)^{k}(2 k+1)\left(\begin{array}{c}
n+1 \\
2 k+1
\end{array}\right) \tan ^{2 k} \phi_{2} .
\end{aligned}
$$

Solving (5.5) for a given value of " $n$," we get $b_{0}$ and $b_{1}$.

THEOREM 5.1. The system of (5.5) possesses a solution, if the difference between " $\Phi_{1} / n$ " and the vertex angle between $L_{1}$ and $L_{2}$ is not an odd integral multiple of " $\pi /(2 n)$."

Proof. Let $z=e^{i \phi_{1}}$. Hence,

$$
\begin{aligned}
z^{n+1}+z^{-(n+1)} & =\left(\cos \phi_{1}+i \sin \phi_{1}\right)^{n+1}+\left(\cos \phi_{1}-i \sin \phi_{1}\right)^{n+1} \\
& =\cos ^{n+1}\left(\left[1+i \tan \phi_{1}\right]^{n+1}+\left[1-i \tan \phi_{1}\right]^{n+1}\right) .
\end{aligned}
$$

Applying the binomial theorem, we obtain

$$
z^{n+1}+z^{-(n+1)}=2\left[\cos ^{n+1} \phi_{1}\right] z_{0,1}
$$

On the other hand,

$$
z^{n+1}+z^{-(n+1)}=2\left[\cos (n+1) \phi_{1}\right]
$$

Equating the right-hand side of (5.8) and (5.9) and solving for $z_{0,1}$, we get

$$
z_{0,1}=\frac{\cos (n+1) \phi_{1}}{\cos ^{n+1} \phi_{1}}
$$




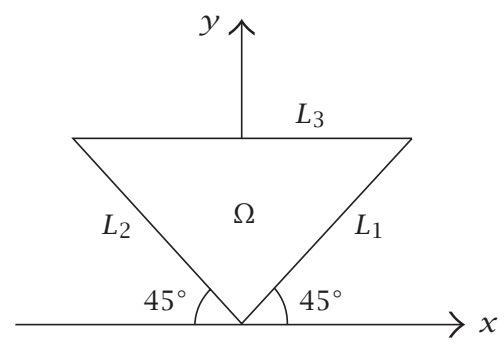

FIGURE 6.1. Geometrical configuration of the problem of Case 6.1.

Similarly, we get

$$
\begin{aligned}
& z_{1,1}=\frac{\sin (n+1) \phi_{1}}{\cos ^{n+1} \phi_{1}}, \\
& z_{0,2}=\frac{(n+1) \sin n \phi_{2}}{\cos ^{n} \phi_{2}}, \\
& z_{1,2}=\frac{(n+1) \cos n \phi_{2}}{\cos ^{n} \phi_{2}} .
\end{aligned}
$$

The system of (5.5) has a unique solution if the determinant

$$
D=\left|\begin{array}{ll}
z_{0,1} & z_{0,2} \\
z_{1,1} & z_{1,2}
\end{array}\right| \neq 0 .
$$

That is,

$$
D=z_{0,1} z_{1,2}-z_{0,2} z_{1,1}=\frac{\cos \left[n\left(\phi_{1}+\phi_{2}\right)+\phi_{1}\right]}{\cos ^{n+1} \phi_{1} \cos ^{n} \phi_{2}}(n+1) \neq 0
$$

which is satisfied if the difference between " $\Phi_{1} / n$ " and the vertex angle between $L_{1}$ and $L_{2}$ is not an odd multiple of " $\pi /(2 n)$."

\section{Special cases}

CASE 6.1. Boundary conditions are combinations of two different degrees of polynomials.

The governing equation for the distribution of temperature $T(x, y)$ is given by

$$
\frac{\partial^{2} T}{\partial x^{2}}+\frac{\partial^{2} T}{\partial y^{2}}=0, \quad(x, y) \in \Omega
$$


with the following boundary conditions:

$$
\begin{aligned}
T(x, y) & =q(x), \quad(x, y) \in L_{1}, \\
\frac{\partial T}{\partial y} & =\beta_{1} x+\beta_{2} x^{5}, \quad(x, y) \in L_{2}, \\
\phi_{1} & =45^{\circ}, \quad \phi_{2}=45^{\circ}, \quad \phi_{3}=0^{\circ} .
\end{aligned}
$$

From the principle of superposition, write

$$
T(x, y)=T_{1}(x, y)+T_{2}(x, y)
$$

where the boundary conditions for $T_{1}(x, y)$ are

$$
\begin{aligned}
T_{1}(x, y) & =\alpha_{1} q_{1}(x), \quad(x, y) \in L_{1}, \\
\frac{\partial T_{1}}{\partial y} & =\beta_{1} x, \quad(x, y) \in L_{2},
\end{aligned}
$$

and the boundary conditions for $T_{2}(x, y)$ are

$$
\begin{aligned}
T_{2}(x, y) & =\alpha_{2} q_{2}(x), \quad(x, y) \in L_{1}, \\
\frac{\partial T_{2}}{\partial y} & =\beta_{2} x^{5}, \quad(x, y) \in L_{2},
\end{aligned}
$$

where

$$
q(x)=\alpha_{1} q_{1}(x)+\alpha_{2} q_{2}(x) .
$$

Setting $n=1$ in the general solution (5.2) and (5.4), we get

$$
\begin{aligned}
T_{1}(x, y) & =\frac{\beta_{1}-\alpha_{1}}{2}\left(x^{2}-y^{2}\right)+\alpha_{1} y x, \\
\left.\frac{\partial T_{1}}{\partial n}(x, y)\right|_{L_{3}} & =b\left(\alpha_{1}-\beta_{1}\right)+\alpha_{1} x .
\end{aligned}
$$

Setting $n=5$ in the general solution (5.2) and (5.4), we get

$$
\begin{aligned}
T_{2}(x, y)= & \frac{3 \alpha_{2}-\beta_{2}}{24}\left(x^{6}-15 y^{2} x^{4}-15 y^{4} x^{2}-y^{6}\right) \\
& -\frac{\alpha_{2}}{8}\left(6 y x^{5}-20 y^{3} x^{3}+6 y^{5} x\right) \\
\left.\frac{\partial T_{2}}{\partial n}(x, y)\right|_{L_{3}}= & -\frac{3 \alpha_{2}-\beta_{2}}{4} b\left(5 x^{4}-10 b^{2} x^{2}+b^{4}\right) \\
& -\frac{3 \alpha_{2}}{4}\left(x^{5}-10 b^{2} x^{3}+5 b^{4} x\right)
\end{aligned}
$$




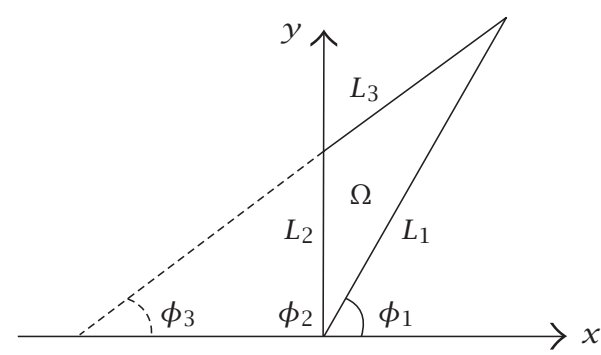

FIGURE 6.2. Geometrical configuration of the problem of Case 6.2.

Hence, the analytic solutions have the form

$$
\begin{aligned}
T(x, y)= & \frac{\beta_{1}-\alpha_{1}}{2}\left(x^{2}-y^{2}\right)+\frac{3 \alpha_{2}-\beta_{2}}{24}\left(x^{6}-15 y^{2} x^{4}-15 y^{4} x^{2}-y^{6}\right) \\
& -\frac{\alpha_{2}}{8}\left(6 y x^{5}-20 y^{3} x^{3}+6 y^{5} x\right), \\
\left.\frac{\partial T}{\partial n}(x, y)\right|_{L_{3}}= & b\left(\alpha_{1}-\beta_{1}\right)+\alpha_{1} x-\frac{3 \alpha_{2}-\beta_{2}}{4} b\left(5 x^{4}-10 b^{2} x^{2}+b^{4}\right) \\
& -\frac{3 \alpha_{2}}{4}\left(x^{5}-10 b^{2} x^{3}+5 b^{4} x\right) .
\end{aligned}
$$

CASE 6.2. One of the boundaries is vertical.

The governing equation for the distribution of temperature $T(x, y)$ is given by

$$
\frac{\partial^{2} T}{\partial x^{2}}+\frac{\partial^{2} T}{\partial y^{2}}=0, \quad(x, y) \in \Omega
$$

with the following boundary conditions:

$$
\begin{aligned}
T(x, y) & =\alpha q(y), \quad(x, y) \in L_{1}, \\
\frac{\partial T}{\partial x} & =\beta y^{n}, \quad(x, y) \in L_{2}, \quad \phi_{2}=90^{\circ} .
\end{aligned}
$$

Write

$$
T(x, y)=w(x, y) q(y), \quad q(y) \neq \equiv 0 \text { in } \Omega,
$$

by which differential equation (6.10) takes the form

$$
q(y)\left(\frac{\partial^{2} w}{\partial x^{2}}+\frac{\partial^{2} w}{\partial y^{2}}\right)+2 \frac{\partial w}{\partial y} \frac{d q}{d y}+w \frac{d^{2} q}{d y^{2}}=0
$$


and the boundary conditions (6.11) take the form

$$
\begin{gathered}
w(x, y)=\alpha, \quad(x, y) \in L_{1}, \\
\frac{\partial w}{\partial x}(x, y)=\frac{\beta y^{n}}{q(y)}, \quad(x, y) \in L_{2} .
\end{gathered}
$$

Applying the invariant analysis, we get

$$
G:\left\{\begin{array}{l}
\bar{x}=C^{x} x, \\
\bar{y}=C^{x} y, \\
\bar{w}=w, \\
\bar{q}=\left[\left(C^{x}\right)^{n+1}\right] q,
\end{array}\right.
$$

and the absolute invariant $\eta(x, y)$ is

$$
\eta(x, y)=\frac{x}{y}
$$

The complete set of absolute invariants corresponding to " $w$ " and " $q$ " are

$$
q(y)=R(y), \quad w(x, y)=F(\eta) .
$$

Substituting (6.16) and (6.17) in (6.13), with $R(y)=y^{n+1}$, we get

$$
\left(\eta^{2}+1\right) \frac{d^{2} F}{d \eta^{2}}-2 n \eta \frac{d F}{d \eta}+n(n+1) F=0
$$

Under the similarity variable $\eta$, the boundary conditions take the form

$$
F\left(\tan \phi_{1}\right)=\alpha, \quad \frac{d F}{d \eta}(0)=\beta .
$$

Solution of (6.18) with the boundary conditions (6.19) is

$$
\begin{aligned}
T(x, y)= & b_{0} \sum_{k=0}^{[(n+1) / 2]}(-1)^{k}\left(\begin{array}{c}
n+1 \\
2 k
\end{array}\right)\left(\frac{x}{y}\right)^{2 k} y^{n+1} \\
& +\left(\frac{b_{1}}{n+1}\right) \sum_{k=0}^{[n / 2]}(-1)^{k}\left(\begin{array}{c}
n+1 \\
2 k+1
\end{array}\right)\left(\frac{x}{y}\right)^{2 k+1} y^{n+1},
\end{aligned}
$$

where

$$
\begin{aligned}
\alpha= & b_{0} \sum_{k=0}^{[(n+1) / 2]}(-1)^{k}\left(\begin{array}{c}
n+1 \\
2 k
\end{array}\right) \cot ^{2 k+1} \phi_{1} \\
& +\left(\frac{b_{1}}{n+1}\right) \sum_{k=0}^{[n / 2]}(-1)^{k}\left(\begin{array}{c}
n+1 \\
2 k+1
\end{array}\right) \cot ^{2 k+1} \phi_{1}, \\
\beta= & b_{1} .
\end{aligned}
$$




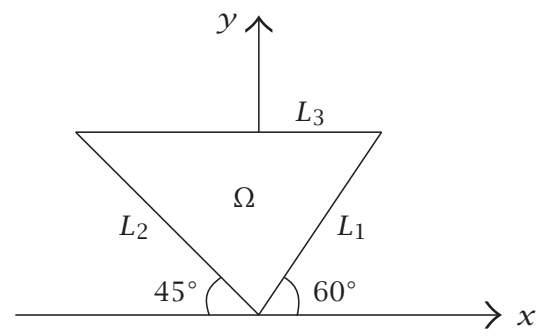

FIGURE 6.3. Geometrical configuration of the problem of Case 6.3.

Solving (6.21) for the given value of " $n$," we get both $b_{0}$ and $b_{1}$.

The heat flux across $L_{3}$ is

$$
\begin{aligned}
\left.\frac{\partial T}{\partial n}(x, y)\right|_{L_{3}}= & b_{0}\left[-\sin \phi_{3} N_{0,1}+\cos \phi_{3} N_{0,2}\right] \\
& +\left(\frac{b_{1}}{n}\right)\left[-\sin \phi_{3} N_{1,1}+\cos \phi_{3} N_{1,2}\right]
\end{aligned}
$$

where

$$
\begin{aligned}
& N_{0,1}=\sum_{k=0}^{[(n+1) / 2]}(-1)^{k}(2 k)\left(\begin{array}{c}
n+1 \\
2 k
\end{array}\right) x^{2 k-1} y^{n-2 k}, \\
& N_{1,1}=\sum_{k=0}^{[n / 2]}(-1)^{k}(2 k+1)\left(\begin{array}{c}
n+1 \\
2 k+1
\end{array}\right) x^{2 k} y^{n-2 k-1}, \\
& N_{0,2}=\sum_{k=1}^{[n / 2]}(-1)^{k}(n-2 k+1)\left(\begin{array}{c}
n+1 \\
2 k
\end{array}\right) x^{2 k} y^{n-2 k}, \\
& N_{1,2}=\sum_{k=0}^{[(n-1) / 2]}(-1)^{k}(n-2 k)\left(\begin{array}{c}
n+1 \\
2 k+1
\end{array}\right) x^{2 k+1} y^{n-2 k+1} .
\end{aligned}
$$

CASE 6.3. The two boundary conditions are identical.

The governing equation for the distribution of temperature $T(x, y)$ is given by

$$
\frac{\partial^{2} T}{\partial x^{2}}+\frac{\partial^{2} T}{\partial y^{2}}=0, \quad(x, y) \in \Omega
$$

with the following boundary conditions:

$$
\begin{aligned}
T(x, y) & =\alpha q(x), \quad(x, y) \in L_{1}, \\
\frac{\partial T}{\partial y} & =-\frac{3}{4} \alpha x^{2}, \quad(x, y) \in L_{2}, \\
\phi_{1} & =60^{\circ}, \quad \phi_{2}=45^{\circ}, \quad \phi_{3}=0^{\circ} .
\end{aligned}
$$


Write

$$
T(x, y)=w(x, y) q(x), \quad q(x) \not \equiv 0 \text { in } \Omega,
$$

by which differential equation (6.24) takes the form

$$
q(x)\left(\frac{\partial^{2} w}{\partial x^{2}}+\frac{\partial^{2} w}{\partial y^{2}}\right)+2 \frac{\partial w}{\partial x} \frac{d q}{d x}+w \frac{d^{2} q}{d x^{2}}=0,
$$

and the boundary conditions (6.11) take the form

$$
\begin{aligned}
w(x, y) & =\alpha, \quad(x, y) \in L_{1} \\
\frac{\partial w}{\partial y}(x, y) & =-\frac{3 \alpha x^{2}}{4 q(x)}, \quad(x, y) \in L_{2} .
\end{aligned}
$$

Applying the invariant analysis, we get

$$
G:\left\{\begin{array}{l}
\bar{x}=C^{x} x, \\
\bar{y}=C^{x} y, \\
\bar{w}=w, \\
\bar{q}=\left[\left(C^{x}\right)^{3}\right] q,
\end{array}\right.
$$

and the absolute invariant $\eta(x, y)$ is

$$
\eta(x, y)=\frac{y}{x} .
$$

The complete set of absolute invariants corresponding to " $w$ " and " $q$ " are

$$
q(x)=R(x), \quad w(x, y)=F(\eta)
$$

Substituting (6.30) and (6.31) in (6.27), with $R(x)=x^{3}$, we get

$$
\left(\eta^{2}+1\right) \frac{d^{2} F}{d \eta^{2}}-4 \eta \frac{d F}{d \eta}+6 F=0 .
$$

Under the similarity variable $\eta$, the boundary conditions take the form

$$
F(\sqrt{3})=\alpha, \quad \frac{d F}{d \eta}(-1)=-\frac{3}{4} \alpha
$$

It can be easily shown that the two conditions (6.33) are identical.

Hence, to find the second condition, assume that

$$
\left.\frac{\partial T}{\partial y}(x, y)\right|_{L_{3}}=\frac{3}{4} \alpha b x+\gamma\left(x^{2}-b^{2}\right),
$$

where " $\gamma$ " is a constant. 
The solution of (6.32) with the aid of the boundary conditions (6.33) and (6.34) is

$$
T(x, y)=-\frac{\alpha}{8}\left(x^{3}-3 x y^{2}\right)+\frac{\gamma}{3}\left(3 x^{2} y-y^{3}\right)
$$

\section{REFERENCES}

[1] M. B. Abd-el-Malek, Group method analysis of nonlinear temperature variation across the lake depth, Proc. 21st International Colloquium on Group Theoretical Methods in Physics Group 21 (Goslar, Germany, 1996), World Scientific, Singapore, 1997, pp. 255-260.

[2] M. B. Abd-el-Malek and N. A. Badran, Group method analysis of unsteady freeconvective laminar boundary-layer flow on a nonisothermal vertical circular cylinder, Acta Mech. 85 (1990), no. 3-4, 193-206.

[3] _ Group method analysis of steady free-convective laminar boundary-layer flow on a nonisothermal vertical circular cylinder, J. Comput. Appl. Math. 36 (1991), no. 2, 227-238.

[4] M. B. Abd-el-Malek, Y. Z. Boutros, and N. A. Badran, Group method analysis of unsteady free-convective laminar boundary-layer flow on a nonisothermal vertical flat plate, J. Engrg. Math. 24 (1990), no. 4, 343-368.

[5] N. A. Badran and M. B. Abd-el-Malek, Group method analysis of the dispersion of gaseous pollutants in the presence of a temperature inversion, Modern Group Analysis: Advanced Analytical and Computational Methods in Mathematical Physics (Acireale, 1992), Kluwer Academic Publishers, Dordrecht, 1993, pp. 35-41.

[6] G. Birkhoff, Mathematics for engineers, Elect. Eng. 67 (1948), 1185.

[7] Y. Z. Boutros, M. B. Abd-el-Malek, I. A. El-Awadi, and S. M. A. El-Mansi, Group method for temperature analysis of thermally stagnant lakes, Acta Mech. 133 (1999), no. 1-4, 131-144.

[8] M. J. Moran and R. A. Gaggioli, Reduction of the number of variables in systems of partial differential equations, with auxiliary conditions, SIAM J. Appl. Math. 16 (1968), 202-215.

[9] A. J. A. Morgan, The reduction by one of the number of independent variables in some systems of partial differential equations, Quart. J. Math. Oxford Ser. (2) 3 (1952), 250-259.

Mina B. Abd-el-Malek: Department of Mathematics, School of Engineering and Sciences, The American University in Cairo, Cairo 11511, Egypt

E-mail address: minab@aucegypt.edu

Fayez H. Michael: Department of Mathematics, Faculty of Science, Alexandria University, Alexandria, Egypt

Samy M. A. El-Mansi: Department of Mathematics, School of Engineering and Sciences, The American University in Cairo, Cairo 11511, Egypt 


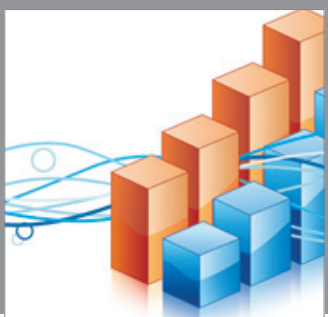

Advances in

Operations Research

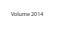

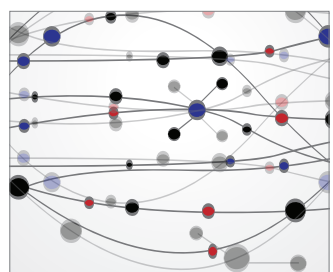

\section{The Scientific} World Journal
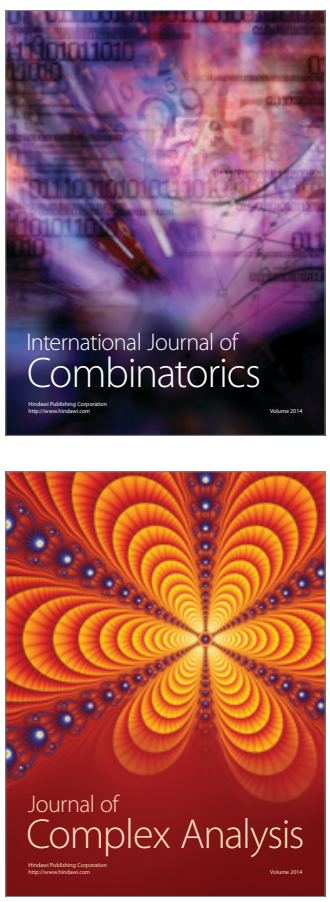

International Journal of

Mathematics and

Mathematical

Sciences
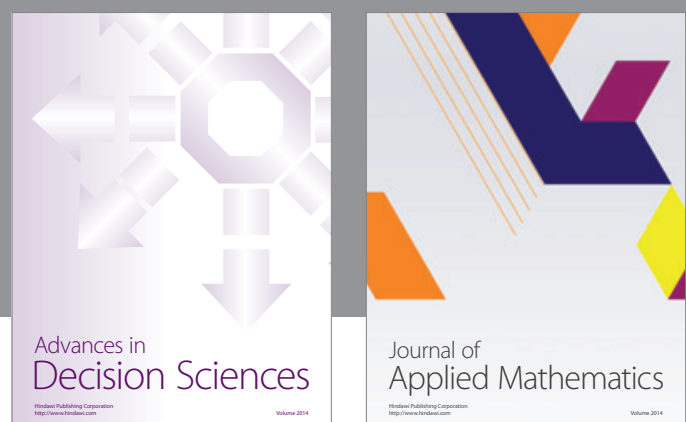

Journal of

Applied Mathematics
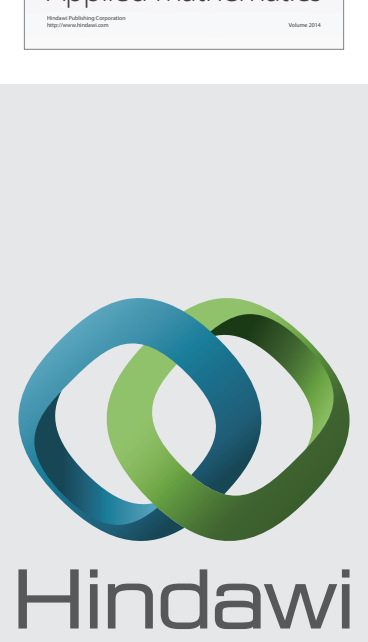

Submit your manuscripts at http://www.hindawi.com
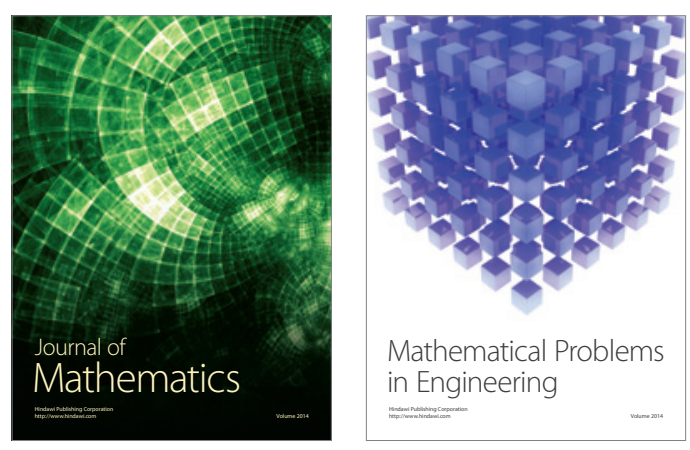

Mathematical Problems in Engineering
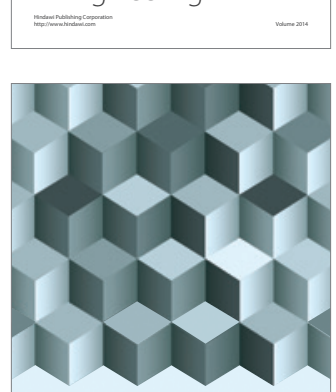

Journal of

Function Spaces
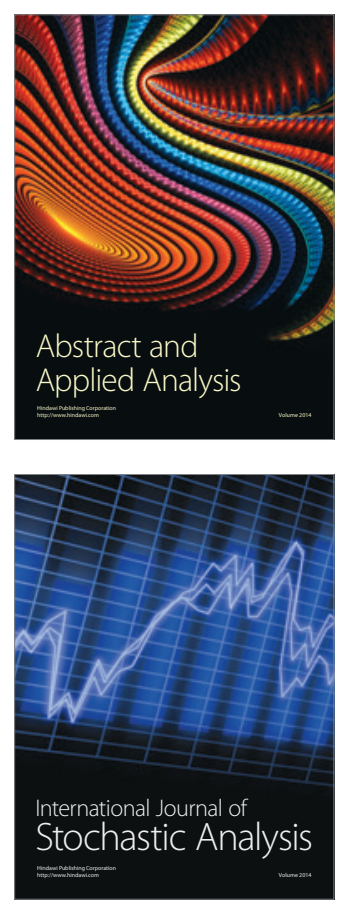

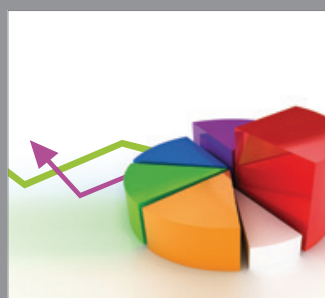

ournal of

Probability and Statistics

Promensencen
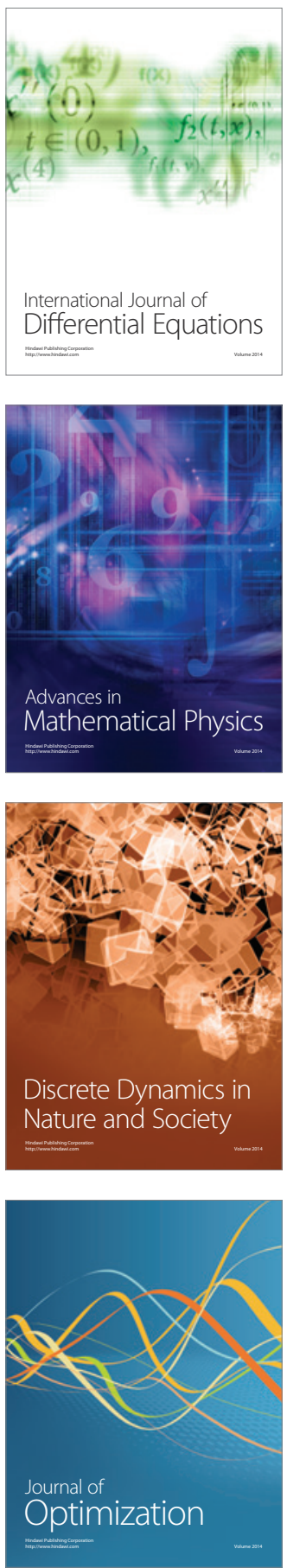